\title{
Resistencia compresiva de dientes con conductos amplios restaurados con dos técnicas
}

\section{Compressive resistance of teeth with large conducts restored with two techniques}

\author{
Johana Sánchez ${ }^{1}$, Andra Jiménez ${ }^{1}$ \\ 1 Fundación Universidad San Martín, Colombia \\ 2 Universidad Metropolitana, Colombia. \\ * Correspondencia autor: Adalgisa Alcocer \\ Calle 76 \# 42 -78 Barranquilla- Colombia. | Telefono \\ +573016506110 | Email: aalcocer@unimetro.edu.co \\ Trabajo recibido el 24/07/2017. Aprobado para su \\ publicación el 22/10/2017
}

\section{RESUMEN}

Objetivo: Comparar la resistencia compresiva de dientes premolares uniradiculares, tratados endodónticamente restaurados mediante la técnica de monobloque y complementación.

Materiales y métodos: Se seleccionaron 45 premolares inferiores unirradiculares, los cuales fueron distribuidos aleatoriamente en 3 grupos, según el diseño reconstructivo: monobloque y complementación; el tercer grupo restaurado bajo la técnica de núcleo colado fue utilizado como grupo control. Para determinar diferencias en la resistencia a la fractura, se realizó un análisis de Varianza (ANOVA a una Vía) y test post hoc de Bonferroni

Resultados: El promedio de la resistencia fue mayor en el grupo dientes rehabilitados con la técnica convencional, seguido de la técnica de monobloque, y en tercer lugar encontramos la técnica de complementación con el valor más bajo. Sin embargo, las técnicas de monobloque y complementación presentan resistencias similares.

Conclusiones: La resistencia a la fractura de dientes restaurados con pernos colados es mayor a la de los restaurados con pernos prefabricados fibras de vidrio con técnicas de complementación y monobloque. Sin embargo, producen mayor cantidad de fallas no reparables o de mal pronóstico que los dientes restaurados con pernos prefabricados de fibras de vidrio por su módulo elástico.

PALABRAS CLAVES

Poste de fibra, Diente endodónticamente tratado, Técnica.

Rev. Clin. Periodoncia Implantol. Rehabil. Oral Vol. 11(1); 20-23, 2018.

\section{ABSTRACT}

Objective: To compare the compressive strength of single-rooted premolar teeth, treated endodontically and restored using the monoblock technique and complementation. Materials and methods: 45 single-rooted inferior premolars were selected, which were randomly distributed in 3 groups, 2 according to the reconstructive design: monoblock and complementation; the third group, restored under the cast core technique, was used as a control group. To determine differences in fracture resistance, we performed an analysis of Variance (One-way ANOVA) and post hoc Bonferroni test

Results: The mean resistance was higher in the group rehabilitated with the conventional technique, followed by the monoblock technique, and thirdly we find the technique of complementation with the lowest value. However, the monoblock and complementation techniques exhibited similar strengths.

Conclusions: Restored teeth with cast posts have a greater resistance to fracture than restorations with prefabricated posts, glass fibers with complementation and monoblock techniques. However, they produce more non-repairable or poor prognostic failures than restored teeth with prefabricated glass fiber posts for their elastic modulus.

KEYWORDS

Fiber post, Endodontically treated tooth, Technique.

Rev. Clin. Periodoncia Implantol. Rehabil. Oral Vol. 11(1); 20-23, 2018. 


\section{INTRODUCCIÓN}

La pérdida de la estructura dental es uno de los grandes inconvenientes para realizar una restauración, planteando un desafío al rehabilitador. Cuando se prepara el canal pulpar para recibir un perno se requiere realizar el desgaste dental el cual ocasiona debilitamiento de la raíz, que a su vez no será sustituida o compensada por la colocación de un perno y dependiendo del perno se pueden generar tensiones internas que conlleven a riesgo de fractura radicular(1). La elección de la técnica adecuada para tratar estas condiciones es un reto para el rehabilitador oral, y muchos prefieren la exodoncia(2).

Los estudios clínicos muestran que hay un porcentaje relativamente alto de fracturas radiculares causadas por los núcleos colados, en conductos dilatados o ampliados $^{(3)}$. Esto puede ocurrir entre otras cosas por el efecto de cuña que produce un retenedor radicular sobre las paredes del conducto(1). Otra hipótesis aceptada es que la fractura puede estar relacionada con las propiedades físicas de los materiales empleados $^{(4)}$. En los casos en que los conductos radiculares se encuentren comprometidos bien sea por la presencia de ápices abiertos, sobre-preparaciones por restauraciones anteriores amplias retenidas por núcleos, caries, fracturas, trastornos congénitos o reabsorción interna, o como sucede en casos de traumas dentales en pacientes jóvenes que frecuentemente interrumpen el cierre apical y desarrollo de la raíz completa, generando la formación de un conducto ensanchado permanente, por lo que la decisión clínica se complica, debido a que los conductos ensanchados son más susceptibles a la fractura, produciendo paredes remanentes delgadas. Lo anterior requiere el uso de técnicas de restauración que no comprometan la integridad de la estructura de la raíz restante ${ }^{(5,6)}$. En esta situación, lo más indicado es rehabilitar con núcleos colados, pero algunos autores ${ }^{(7-10)}$ han sugerido las técnicas de complementación y monobloque como alternativa conservadora y estética en estos casos

El objetivo de este estudio es comparar la resistencia compresiva de dientes premolares uniradiculares, tratados endodónticamente restaurados mediante la técnica de monobloque y complementación.

\section{MATERIALES Y MÉTODOS}

Se desarrolló un estudio experimental in vitro. La muestra estuvo conformada por 45 dientes premolares inferiores uniradiculares sanos, sin caries, lesiones pulpares o dilaceraciones radiculares; medida coronal a apical $22 \mathrm{~mm} \pm 1 \mathrm{~mm}$, sin conductos accesorios, extraídos como parte de tratamiento ortodóntico, fueron conservados en solución salina estéril, seguido de la remoción de cálculos y limpieza radicular ${ }^{(11)}$.

Se conformaron aleatoriamente 3 grupos de 15 dientes: grupo A: Técnica de Monobloque (tratamiento con poste de fibra de vidrio y relleno resinoso); grupo B: Técnica de complementación (tratamiento con Postes de fibra de vidrio con reconstrucción del muñón); grupo C: Técnica de Núcleo colado en metal base níquel-cromo, aleación escogida para la realización debido a su maleabilidad y ductilidad.

Se realizó corte coronal $2 \mathrm{~mm}$ de la línea amelocementaria con disco diamantado (KG Sorensen $囚$ ) un disco por cada 5 dientes, luego tratamiento endodóntico con sistema rotatorio Pro Taper universal ${ }^{\circledR}$; se buscó lima apical principal F4 correspondiente a ISO \#40, acompañado con irrigación con hipoclorito de $\mathrm{Na}$ al $5 \%$, la técnica de obturación fue "condensación lateral", y se obturaron los conductos con cemento (Topseal() por el mismo operador; posteriormente se realizó la desobturación inmediata terminado el tratamiento de conducto, dejando $5 \mathrm{~mm}$ de gutapercha de selle apical, con fresas de pesso No 3 (VDW®), 3 fresas por grupo.

Se realizó montaje de los dientes en cubo de resina acrílica transparente autopolimerizable, (Veracril@) de $2 \times 1,8 \mathrm{~cm}$ en posición central. Luego se debilitaron las paredes dentinales del conducto radicular hasta conseguir $1 \mathrm{~mm}$ de espesor a nivel del tercio cervical a los dientes de los 3 grupos, con una fresa de talla grano estándar Diatech 856 (una por cada 5 dientes). El grosor de las paredes fue comprobado con un calibrador a nivel cervical.

Se realizó la preparación de los conductos para recibir los postes de fibra de vidrio con fresas específicas de la misma marca de los postes en fibra de vidrio (Tenax Fiber post trans ${ }^{\circledR}$ ); todos los dientes se prepararon hasta que consiguieran un diámetro de $1,5 \mathrm{~mm}$ como el del poste principal y para los postes accesorios en diámetro fue de $1,1 \mathrm{~mm}$. Se inició el procedimiento con el grupo A bajo la técnica de monobloque, colocándose el poste adentro del conducto y se observó que este oscilara en sentido mesio-distal y vestíbulo-lingual. Se agregó glicerina líquida en el interior del conducto (A paredes) utilizando micro pincel. El poste y el conducto fueron desinfectados con hipoclorito de $\mathrm{Na}$ al $5 \%$ por $2 \mathrm{~min}$ y se lavó con suero fisiológico y se secó con una gasa estéril. Se aplicó el silano (Ultradent products Inc®) y se esperó $1 \mathrm{~min}$. Se colocó alrededor del poste una cantidad ParaCore $®$ (según indicaciones del fabricante), se colocaron en la luz del conducto radicular, se aplicó una ligera presión constante y sostenida hasta lograr percibir al tacto la resistencia ofrecida por la paredes del conducto, se retiró el exceso de material con una espátula y se fotoactivo por 5 seg con lámpara de fotocurado Blue Phase G2®, después de haber polimerizado por 5 segundos como máximo, se retiró el poste y se terminó de fotocurar afuera durante 20 seg por cada superficie. Se rectificó la adaptación en el conducto del poste anatómico y se terminó la conformación del muñón utilizando forma plástica No.5 contenida en el kit de ParaCore $®$, y se eliminó la glicerina de la superficie del espécimen usando una gasa estéril embebida de alcohol etílico; se colocó el agente cementante resinoso dual Relix U200® en la parte interna del conducto radicular y se introdujo el poste anatómico, se fotoactivó, se eliminaron los excesos y luego un fotoactivado final por 40 seg por cada superficie.

En cuanto al grupo B con la técnica de Complementación, inició con igual protocolo mecánico y de desinfección, excluyendo el aislamiento del conducto con glicerina líquida; se usó el sistema adhesivo OneCoat Bond (SL $®$ componente A + componente B) sobre los postes usando un micro pincel, se aplicó aire por $5 \mathrm{~s}$ y se fotoactivó usando lámpara

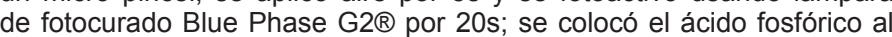
$35 \%$ Gel (Etchant $\left.{ }^{\circledR}\right)$ sobre la superficie de la dentina radicular, se lavó y se secó con puntas de papel, se aplicó el sistema adhesivo OneCoat Bond ( $\mathrm{SL} \otimes$ componentes $\mathrm{A}+\mathrm{B}$ ) usando un micro pincel friccionando la superficie de la dentina radicular. Se eliminan los excesos con aire por $5 \mathrm{~s}$; se colocó alrededor del poste principal una cantidad ParaCore $®$ (mezclando el catalizador y base según indicaciones del fabricante). En estas condiciones se encontraban listos para poder ser colocados en la luz del conducto radicular, a la inserción del poste con el ParaCore $\AA ;$ se aplicó una ligera presión constante y sostenida, luego se le adicionó el poste accesorio aplicando también un poco de presión, se eliminaron los excesos y luego se fotoactivó $40 \mathrm{~s}$, por superficie, siguiendo las recomendaciones sugeridas por el fabricante, seguido de esto, se terminó la conformación del muñón utilizando forma plástica No.5 contenida en el kit de ParaCore $®$, para garantizar la uniformidad de los mismos entre cada espécimen, y se fotocuró por 40 seg por cada superficie.

El grupo C fue utilizado como control, conformado por dientes restaurados con núcleos colados en metal base VeraBond® para Vita 2 a base de níquel-cromo. En este se inició con el aislamiento del conducto con petrolato líquido, el cual se aplica con un micro-pincel sobre las paredes de éste; seguido de ello se procedió a realizar el patrón de núcleo con duralpin y resina acrílica Resin74@ conformando el muñón, fue tallado posteriormente a su polimerización con fresas de tallado de diamante procurando mantener la misma forma y dimensión de los muñones del grupo A y B en cuanto altura e inclinación de los planos cuspídeos.

Se enviaron al laboratorio los patrones para ser colados en metal base y se eliminó el exceso de petrolato en el conducto con un micro-pincel que contenía alcohol etílico al $95 \%$ y luego se lavó con suero fisiológico y se secó. Los núcleos fueron cementados con Relix U200®, siguiendo todas las especificaciones del fabricante.

Se fabricó un dispositivo en acero al carbón y aluminio para la colocación de los especímenes en una angulación de $45^{\circ}$ con una concavidad de $2 \times 1,8 \mathrm{~cm}$ para la posterior realización de las pruebas, basado en la angulación ideal para ensayos In vitro descrito por Moyers ${ }^{(12)}$, para garantizar que la ubicación de los especímenes al momento de la aplicación de la carga fuera la misma, además de una punta plana con un diámetro de $2 \mathrm{~mm}$ en la superficie de contacto en el mismo material.

En una máquina de ensayos universal SHIMADZU UH-100ª con rango de $0-100 \mathrm{KN}$, se sometieron los especímenes a una carga compresiva continua a un ángulo de $45^{\circ}$ con una velocidad de $2,0 \mathrm{~mm} / \mathrm{min}$ con una carga de $10 \mathrm{Kg} / \mathrm{F}$ hasta obtener el primer evento de fractura. Se tomó como definición de fractura el punto en el que se observó una caída brusca e instantánea mayor que el $25 \%$ de la carga aplicada. Para la mayoría de las muestras se escuchó un "crack" (ruido). La prueba se terminó en este punto y se registró la fuerza en Kilo Newton (KN).

Para el análisis de los sitios de fractura se delimitaron los tercios radiculares y coronales y se colocaron los dientes en azul de metileno, posteriormente se verificó la línea de fractura, por medio de sección en corte sagital con discos diamantados (KG Sorensen $®$ ) en cada uno de los especímenes, identificando en qué tercio ocurrió la falla.

Los resultados fueron tabulados y analizados en el programa SPSS versión 20. Se utilizaron medias de tendencia central (media, moda, mediana) y de dispersión (desviación estándar), se verificó el supuesto de normalidad con la prueba Shapiro Wilk y el supuesto de homocedasticidad se utilizó el test de Levane, a los datos se les realizo transformación logarítmica para corregir la falta del cumplimiento del supuesto de homocedasticidad. Para determinar diferencias en la 
resistencia a la fractura, se realizó ANOVA a una vía y prueba post hoc test de Bonferroni, con un nivel de significancia de 0,05. En todo momento hubo acogimiento de los principios éticos de la declaración de Helsinki y a las normas de Buenas Prácticas Clínicas en Investigación.

\section{RESULTADOS}

El promedio de la resistencia fue mayor en el grupo dientes rehabilitados con la técnica convencional $(463,67+/-239,99 \mathrm{Mpa})$; el promedio de la resistencia de los dientes restaurados con la técnica de complementación fue de 143,06 +/- 52,81Mpa. El promedio de la resistencia de los dientes restaurados con la técnica de monobloque fue de 137,73 +/-63,84Mpa. (Tabla 1)

Al comparar las medias de las resistencias de los dientes tratados con las diferentes técnicas se encontró que existen diferencias estadísticamente significativas $\mathrm{p}<0,05$ entre los promedios de la resistencia de los dientes tratados con las diferentes técnicas. (Tabla 2)

Las diferencias significativas encontradas en las medias se dieron entre la técnica convencional y las otras técnicas $(p<0,05)$; sin embargo, las técnicas de monobloque y complementación presentan resistencias similares $(p>0,05)$. No hay suficiente evidencia para afirmar que el uso de postes complementarios aumenta la resistencia de dientes con conductos amplios rehabilitados al compararlo con la técnica de monobloque (Tabla 3).

Tabla 1. Análisis descriptivo de la Resistencia de dientes(Mpa) con Conductos amplios según tipo de técnica utilizada

\begin{tabular}{|c|c|c|c|c|c|c|c|}
\hline \multirow{2}{*}{ Técnica } & \multirow{2}{*}{ Media } & \multicolumn{2}{|c|}{$\begin{array}{l}\text { Intervalo de confianza para la } \\
\text { media al } 95 \%\end{array}$} & \multirow{2}{*}{ Mediana } & \multirow{2}{*}{ Desv. típ. } & \multirow{2}{*}{ Mínimo } & \multirow{2}{*}{ Máximo } \\
\hline & & $\begin{array}{l}\text { Límite } \\
\text { inferior }\end{array}$ & $\begin{array}{l}\text { Límite } \\
\text { superior }\end{array}$ & & & & \\
\hline T. Complementación & 143,06 & 113,81 & 172,30 & 132,53 & 52,81 & 59,32 & 269,25 \\
\hline T. Monobloque & 137,73 & 102,38 & 173,08 & 136,20 & 63,84 & 43,09 & 275,78 \\
\hline $\begin{array}{l}\text { T. Convencional } \\
\text { (núcleos colados) }\end{array}$ & 463,67 & 330,76 & 596,57 & 416,25 & 239,99 & 125,32 & 1044,30 \\
\hline
\end{tabular}

Tabla 2. Prueba Anova para diferencia de medias

\begin{tabular}{ccccc}
\hline & Suma de cuadrados & gl & Media cuadrática & F \\
\hline Inter-grupos & 13.179 & 2 & 6.589 & 26.640 \\
Intra-grupos & 10.389 & 42 & .247 & .000 \\
Total & 23.568 & 44 & & \\
\hline
\end{tabular}

Tabla 3. Resultados Prueba post hoc Bonferroni

\begin{tabular}{|c|c|c|c|c|c|c|}
\hline \multirow{2}{*}{ (I) Factor } & \multirow{2}{*}{ (J) Factor } & \multirow{2}{*}{$\begin{array}{l}\text { Diferencia de } \\
\text { medias (I-J) }\end{array}$} & \multirow{2}{*}{ Error típico } & \multirow{2}{*}{ p-Valor } & \multicolumn{2}{|c|}{ Intervalo de confianza al $95 \%$} \\
\hline & & & & & Límite inferior & Límite superior \\
\hline \multirow{3}{*}{ Convenciona } & Monobloque & $1.18916^{*}$ & .18160 & .000 & .7363 & 1.6420 \\
\hline & & & & & & \\
\hline & Complementación & $1.10185^{*}$ & .18160 & .000 & .6490 & 1.5547 \\
\hline \multirow{3}{*}{ Monobloque } & Convencional & $-1.18916^{*}$ & .18160 & .000 & -1.6420 & -.7363 \\
\hline & & & & & & \\
\hline & Complementación & -.08731 & .18160 & 1.000 & -.5402 & .3655 \\
\hline \multirow{3}{*}{ Complementació } & Convencional & $-1.10185^{*}$ & .18160 & .000 & -1.5547 & -.6490 \\
\hline & & & & & & \\
\hline & Monobloque & .08731 & .18160 & 1.000 & -.3655 & .5402 \\
\hline
\end{tabular}

Teniendo en cuenta el tipo de fractura se encontró que en el grupo de los dientes rehabilitados con la técnica de complementación el 6,7\% presentó fractura vertical, el $20 \%$ fractura oblicua y el $73.3 \%$ fractura horizontal. En el grupo de dientes rehabilitados con técnica monobloque el $13,3 \%$ presentó fractura vertical, el $26,7 \%$ fractura oblicua y el $53,3 \%$ fractura horizontal. En el grupo de los dientes rehabilitados con la técnica convencional (núcleo colado) el $46,7 \%$ presentó fractura vertical, el $0,0 \%$ fractura oblicua y el $66,7 \%$ fractura horizontal.

Según la ubicación de la fractura se encontró que en el grupo de dientes rehabilitados con la técnica de complementación el 0,0\% presentó fractura de la restauración, el $80 \%$ fractura del tercio coronal y el $20 \%$ fractura del tercio medio. En el grupo de dientes rehabilitados con la técnica de monobloque el $6,7 \%$ presentó fractura de la restauración, el $87 \%$ fractura del tercio coronal y el $7 \%$ fractura del tercio medio. En el grupo de dientes rehabilitados con la técnica convencional (núcleo colado) el $0,0 \%$ presentó fractura de la restauración, el $7 \%$ fractura del tercio coronal y el $93 \%$ fractura del tercio medio.

\section{DISCUSIÓN}

La necesidad de preservación de las piezas dentales ha generado el uso de restauraciones con excelentes propiedades mecánicas, con técnicas y materiales de comportamientos favorables que permiten el éxito clínico, aunque muchas de estas no tienen suficiente evidencia. Sin 
embargo, las cargas nocivas ponen a prueba el éxito de la rehabilitación de dientes debilitados radicularmente. Ligado a esto, el tipo de material utilizado es una de las propiedades que influye en la resistencia a la fractura del diente restaurado y su pronóstico; su rigidez o su módulo de elasticidad se relacionan con la distribución de cargas a través de la estructura dentaria ${ }^{(13)}$. Se encontró que la rehabilitación con técnica de monobloque y complementación con materiales que tienen módulos de elasticidad similares al de la dentina, tienen más bajas concentraciones de esfuerzos que los núcleos colados y los distribuyen de manera diferente que el poste más rígido, comprobando que el material más rígido, el núcleo colado, presenta un alto valor de resistencia y absorbe mayor fuerza (463,67 Mpa).

En el poste de fibra de vidrio bajo dos técnicas de restauración, la complementación presentó un valor de resistencia de 143,06 Mpa y la técnica de monobloque con un valor inferior (137,73 Mpa) recibe un esfuerzo menor, coincidiendo con lo dicho por Omiri y Wahadni ${ }^{(14)}$, quienes recomiendan el uso de postes de fibra de vidrio debido a que además de generar un menor desgaste de la estructura dental remanente, tienen un comportamiento biomecánicamente adecuado; ya que su módulo elástico es similar al de la estructura dentaria, lo que aumenta la posibilidad de mejoramiento de resultados estéticos que son una de las grandes ventajas a la hora de su elección.

Además, Naumann y cols. ${ }^{(15)}$, plantean que el hecho de tener postes con un módulo de elasticidad alto como un retenedor colado, conlleva a que el esfuerzo se distribuya a través del mismo poste, el cual resiste más la deformación. Qing y cols. ${ }^{(16)}$, encontraron diferencias en la resistencia a la fractura en los dos grupos evaluados, planteando que los postes rígidos podrían mejorar la resistencia a la deflexión, confirmando que el poste y muñón colado fueron más resistentes a las fuerzas de deflexión y que mostraban fallas con carga muy altas, aclarando que estos resultados no son prueba para decir que no es conveniente el uso clínico de los postes.

En este estudio, se encontró que existen diferencias estadísticamente significativas $p<0,05$ entre los promedios de la resistencia de dichos dientes restaurados de forma distinta. Es importante tener en cuenta que cuando se aumentan los esfuerzos, en condiciones normales intraoralmente se incrementan en el ligamento periodontal, el cual absorbe una cantidad de esfuerzo y minimiza los efectos sobre el diente y sus estructuras, por lo tanto, estudios que no simulen el ligamento periodontal y su comportamiento, tendrán una variación significativa. Sin embargo, Dejak y cols. ${ }^{(17)}$, encuentran resultados y conclusiones muy similares, pues bajo cargas fisiológicas, los postes hechos en metal o en fibra de vidrio, no comprometen el daño al diente y los postes colados transmiten menos esfuerzo a la dentina, debido a sus propiedades mecánicas de un módulo de elasticidad más alto. Varvara y cols.(18), en un estudio in vitro, hecho para evaluar la resistencia a la fractura de dientes tratados endodónticamente con diferentes alturas de dentina residual, concluyendo que, ante altas cargas, los postes con alto módulo de elasticidad resistieron más las cargas que los postes más flexibles, pero estos últimos presentaron fallas más favorables que los primeros pero concluyeron que estas cargas con fallas catastróficas de los colados se dan a fuerzas que generalmente no se presentan en la cavidad oral, coherente a lo encontrado en el presente estudio.

Con respecto al tipo de fractura, se evidenció que la fractura vertical es más frecuente en la técnica convencional y más baja en la técnica de complementación, la fractura horizontal predominó en el grupo de complementación, a diferencia de las oblicuas donde la técnica de monobloque obtuvo el mayor número de casos. Estudios anteriores informaron que las colocaciones de postes de fibra en los conductos radiculares preparados los hace menos propensos a causar fracturas radiculares verticales en comparación con postes de acero inoxidable ${ }^{(19,20)}$. Algunos mostraron que cuando se produjeron fallos, el tipo de fractura suele repararse con un poste de fibra ${ }^{(21-23)}$, similar a lo encontrado en este estudio donde la fractura más común ocurrió el grupo de dientes restaurados con núcleos colados. Aunque estadísticamente no se encontró diferencia significativa entre los resultados obtenidos entre el comportamiento In vitro de las técnicas de Monobloque y complementación, el manejo clínico para la aplicación de estas técnicas podría decidirse considerando el ahorro de tiempo, material, habilidad del operador y fatiga del paciente.

Una limitación de este estudio es el hecho que se realiza In vitro y los resultados deben extrapolarse directamente a las situaciones clínicas. Nuevos estudios deberían incorporar termociclado.

\section{CONFLICTO DE INTERÉS Y FUENTE DE FINANCIAMIENTO}

Declaramos no tener conflicto de intereses; el presente trabajo fue autofinanciado.

\section{RELEVANCIA CLÍNICA}

Realizar un aporte a la controversia clínica relacionada con las formas de restauración de dientes con conductos amplios, por cuanto el mejoramiento de las condiciones intraradiculares podrá asegurar mayor éxito en la reconstrucción dental, asegurando tanto una mayor funcionalidad como una estética adecuada. Por ello, se consideró importante la búsqueda de la técnica con mejor comportamiento de acuerdo al uso de nuevos materiales y procedimientos ante la aplicación de fuerzas compresivas.

\section{Bibliografía}

1. Yoldas $O$, Akova T, Uysal $H$. An experimental analisys of stresses in simulated flared root canals subjected to various pos-core applications. J Oral Rehabil. 2005 32 (6):427-432

2. Aggarwal V, Singla M, Miglani S, Kohli S. Comparative evaluation of fracture resistance of structurally compromised canals restored with different dowel methods J Prosthond 2012; 21(2):312 - 6.

3. McLaren J, McLaren C, Yaman P, Bin-Shuwaish M, Dennison J, McDonald N. The effect of post type and length on the fracture resistance of endodontically treated teeth. J Prosthet Dent. 2009;101(3):174-82.

4. Lynch C, Burke F. Incomplete tooth fracture following root-canal treatment: a case report. Int Endod J.2002; 35(7):642-44

5 . Kimmel S. Restauration of endodontically treated tooth containing wide o flared canal. NY State Dent J. 2000; 66:36-4

6. Zogheib L, Pereira J, Valle A, Oliveira J, Pegoraro L. Fracture resistance of weakened roots restored with composite resin and glass fiber post: Braz Dent $\mathrm{J}$. 2008:19(4):329-33

7. Grandini S, Goraci C. Clinical evaluation of the use of the fiber post and direct resin restorations for endodontically Treated teeth, Int J Prosthodont. 2005; 18:399-404. 8. Kina S, Bruguera A. Invisible: restauraciones estéticas cerámicas; Sao Paulo: Editorial Dental Press. 2008

9. Clavijo V, Souza N, Ferrarezi M, Susin A. Pinos anatômicos: uma nova perspectiva clínica. Rev Dental Press Estét. 2006; 3(3):100-21.

10. Lamas-Lara C, Jiménez-Castro J, Angulo de la Vega G. Poste anatómico û reporte de caso. Kiru. 2014; 11(1):81-5.

11. Canalda C, Aguadé B. Endodóncia. Técnicas clínicas y bases científicas Barcelona: Masson, S.A. 2001

12. Marchi G, Paulillo L, Pimenta L. Effect of different filling materials in combination with intraradicular posts on the resistance to fracture of weakened roots. J Oral Rehabil. 2003. 30; 623-9

13. Zarone F, Sorrentino R, Apicella D, Valentino B, Ferrari M, Aversa R. Et al. Evaluation of the biomechanical behavior of maxillary central incisors restored by means of endocrowns compared to a natural tooth: a 3D static linear finite elements analysis. Dent Mater. 2006;22(11):1035-44
14. AL-Omiri M, AL-Wahadni A. An ex vivo study of the effects of retained coronal dentine on the strength of teeth restored with composite core and different post and core systems. Int Endod J. 2006; 39(11): 890-9.

15. Naumman M, Preuss A, Frankenberger R. Reinforcement effect of adhesively luted fiber reinforced composite versus titanium post. Dent Mater 2007; 23(2):138-44 16. Qing H, Zhu Z, Chao Y, Zhang WQ. In vitro evaluation of the fracture resistance of anterior endodontically treated teeth restored with glass fiber and zircon posts. $J$ Prosthet Dent 2007; 97(2):93-8.

17. Dejak B, Andrzej M. Finite element analysis of strength and adhesion of cast posts compared to glass fiber-reinforced composite resin posts in anterior teeth. $J$ Prosthet Dent 2011; 105: 115-126.

18. Varvara G, Perinneti G, Dilorio D, Murmura G, Caputi S. In vitro evaluation of fracture resistance and failure mode of internally restored endodontically treated maxillary incisors with differing heights of residual dentin. J Prosthet Dent. 2007; 98: 365-332.

19. Asmussen E, Peatzleldt A, Heitmann T. Stiffness, elastic limit, and strength of newer types of endodontic posts. Dent J. 1999; $27: 275-8$.

20. Pegoretti A, Fanbri I, Zappini G, Bianchetti M. Finite element analysis of a glass fiber reinforced composite endodontic post. Biomaterials 2002; 23: 2667-82

21. Salameh Z, R Sorrentino, Papachini F, Ounsi HF, Tashkandi E, Ferrari M. Fracture resistance and failure patterns of endodontically treated mandibular molars restored using resin composite with or without translucent glass fiber posts. J Endod. $2006 ; 32: 752-5$

22. Eliasson S, J Bergstrom, Sanda A. Periodontal bone loss of teeth with metal posts. J Clin Periodontol 1995; 22:850-3

23. Kurthukoti AJ, Paul J, Gandhi K, Rao DB. Fracture resistance of endodontically treated permanet anterior teeth restores with three different esthetic post systems: An In vitro study. J indian Soc Pedod Prev Dent. 2015; 33:296-301 